\title{
RETRACTED ARTICLE: Recent studies in CFD modelling of pollutant dispersion in street canyons
}

\section{Bodhisatta Hajra (凹)}

Department of Building, Civil and Environmental Engineering, Concordia University, Montreal, Canada H3G 1M8

\section{Article History}

Received: 10 June 2011; Revised: 14 August 2011; Accepted: 15 August 2011

๑ Tsinghua University Press and Springer-Verlag Berlin Heidelberg 2012

This article has been retracted due to disputed authorship.

\footnotetext{
E-mail: b_hajra@encs.concordia.ca
} 